\title{
Dinámica metapoblacional, ocupación espacial y migración en bivalvos de importancia comercial: el caso de la almeja Tawera gayi (Bivalvia: Veneridae) en el Mar Interior de Chiloé
}

\author{
Metapopulation dynamics, spatial occupation and migration \\ of the commercial importance bivalves: the case of the clam \\ Tawera gayi (Bivalvia: Veneridae) from the Chiloé inner sea
}

Luis Figueroa-Fábrega ${ }^{1}$, Javiera Bravo-Samaha ${ }^{2}$, Rodrigo Silva-Haun ${ }^{3} \&$ Tiare Padilla ${ }^{4}$

\section{Resumen}

Tawera gayi (Hupe, 1854), es un molusco bivalvo conocido comúnmente como Juliana, cuya extracción comercial en Chile se concentra tanto en la zona norte del mar interior de Chiloé cómo en las inmediaciones de las islas Desertores. Según las estadísticas pesqueras, los desembarques han disminuido progresivamente en el último tiempo, para lo cual se han esgrimido razones relacionadas tanto con el mercado, la sobreexplotación del recurso y a la no inclusión de la conectividad espacial de los distintos bancos locales en el manejo pesquero. Por lo que el estudio de la dinámica de metapoblaciones (i.e. la variación temporal de la ocupación de los parches) puede ser una herramienta útil para comprender los patrones de abundancia de aquellas especies que presentan una alta conectividad espacial. En el presente trabajo se realizaron simulaciones para dos escenarios descritos en la zona, para dar respuesta a una serie de interrogantes relacionadas con la conectividad espacial entre bancos, los efectos de la disminución en la conectividad y el grado de incidencia de algunos de ellos. Los resultados nos indican que, al disminuir la disponibilidad de larvas por perdida de la fracción reproductiva, se reduce la probabilidad de colonización, afectando además la probabilidad de dispersión y la incidencia entre bancos cambiando la dinámica de la metapoblación. En una perspectiva temporal, se constato que la población de Juliana paso de tener una alta conectividad entre los bancos a una condición de auto-reclutamiento de algunos bancos particulares de mayor tamaño, tal como se ha visto en otras almejas de importancia comercial. Por consiguiente, es fundamental considerar la conectividad espacial en el manejo pesquero, considerando además los efectos sinérgicos que la extracción de especies de interés comercial pueda generar en los ecosistemas marinos del mar interior de Chiloé.

\section{Palabras clave:}

Dinámica de ocupación-extinción, dinámica de paisaje, Modelo SPOMs, Modelo de Incidencia.

\section{Abstract}

Tawera gayi (Hupe, 1854), is a bivalve mollusk commonly known as Juliana, whose commercial extraction in Chile is concentrated both in the northern area of the Chiloé inner sea and in the vicinity of the Desertores Islands. According to fishery statistics, landings have progressively decreased in recent times, for which reasons have been raised related to the market and the overexploitation of the resource and the non-inclusion of the spatial connectivity of

\footnotetext{
1 Departamento de Oceanografía y Medioambiente. Instituto de Fomento Pesquero. Blanco 839, Valparaíso, Chile. $\$ luis.figueroa@ifop.cl

2 Facultad de Ecología y Recursos Naturales, Universidad Andrés Bello, Republica 440, Santiago, Chile. j.bravosamaha@gmail.com

3 Escuela de Ingeniería, Universidad Viña del Mar, Agua Santa 7055, Viña del Mar, Chile. rsilva@uvm.cl

4 Biología Marina, Universidad de Valparaíso. Avenida Borgoño 16344, Viña del Mar. tiare.padilla.zambrano@ gmail.com
} 
the various local patches in fisheries management. For this reason, the study of the metapopulation dynamics (ie the temporal variation of the occupation of the patches), can be a useful tool to understand species that seem to have a high spatial connectivity. In the present work simulations were carried out for two scenarios described in the area to answer a series of questions related to the spatial connectivity between patches, the effects of the decrease of patches for connectivity and the degree of incidence of some local population in the Chiloé inner sea. The results indicate that, by reducing the availability of larvae due to loss of the reproductive fraction, the probability of colonization is reduced, also affecting the probability of dispersion and the incidence among patches. This is summarized in a change in the dynamics of the metapopulation. In a temporal perspective, it was found that the population of Juliana went from having a high connectivity among the banks to a condition of self-recruitment of some larger patches, as seen in other clams of commercial importance. Therefore, it is essential to consider the spatial connectivity in fisheries management, considering also the synergistic effects that the extraction of species of commercial interest can generate in the marine ecosystems of the Chiloé inner sea.

\section{Key words:}

Occupancy-extinction dynamics, SPOMS, Landscape dynamics, Incidence function model.

\section{INTRODUCCIÓN}

Los procesos que están detrás de la variabilidad en las abundancias bivalvos de fondos blandos han sido discutidos ampliamente por la comunidad científica (eg. Rodríguez et al. 1993; Kininmonth et al. 2011; Zhang et al. 2015), existiendo un consenso en que tanto la intensidad del asentamiento de las larvas como la mortalidad y el crecimiento posterior al asentamiento son los procesos que subyacen a estos cambios poblacionales (Snelgrove \& Butman, 1994). La búsqueda de patrones en la dispersión de larvas entre los parches o poblaciones locales de los bivalvos bentónicos es una herramienta fundamental para comprender la conectividad y la persistencia de las poblaciones (Botsford, 2001; Pineda et al. 2007).
El modelo simple de metapoblación propuesto por Levins (1969) considera la conectividad entre unidades discretas o parches de una población, como uno de los ejes centrales de la dinámica poblacional, ya que el equilibrio existente entre la extinción y recolonización de poblaciones locales permite la permanencia a largo plazo de la especie en cuestión (Sale et al. 2006). Por tal motivo el estudio de la dinámica de metapoblaciones (i.e. la variación temporal de la ocupación de los parches (Mestre et al. 2016a)), parece ser la manera apropiada para comprender la alta variabilidad de especies que forman metapoblaciones.

Si consideramos que la mayoría de los bivalvos marinos presentan ciclos de vida que incluyen un período larval dispersivo, seguido por etapas de individuos juveniles y adultos sésiles (Caddy \& Defeo, 2003), es esperable que la escala espacial juegue un rol preponderante en la sobrevivencia de los bivalvos marinos. Ante esto, tanto Hanski y Gilpin (1991) y Sale et al. (2006) han sugerido que estas especies puedan ser consideradas como verdaderas "Metapoblaciones", identificando la existencia de poblaciones o bancos locales centrales denominados "Fuente o Madre", los cuales funcionan como fuente principal de emigrantes; y poblaciones o bancos "Satélites o Sumideros", los cuales reciben a estos individuos migrantes y que están sujetas a la dinámica de extinción y re-colonización local (Hanski, 1994; Hanski \& Gilpin, 1997; Hanski \& Gaggiotti, 2004).

Tawera gayi (Hupe fide Gay 1854), es un molusco bivalvo conocido comúnmente como Juliana, Almeja Fina Chilena o Baby Clam (Osorio, 2002). Su distribución geográfica en el Pacifico Sur abarca desde Valparaíso $\left(33^{\circ} \mathrm{S}\right)$ hasta el Canal Beagle (i.e. $54^{\circ}-55^{\circ}$ S) (Dell, 1974; Soot-Ryen, 1959) y su extracción comercial se concentra tanto en la zona norte del Mar Interior de Chiloé $\left(42,0^{\circ} \mathrm{S}\right.$ - 73,0 $\left.0^{\circ} \mathrm{O}\right)$, cómo en las inmediaciones de las Islas Desertores $\left(42,7^{\circ} \mathrm{S}-72,0^{\circ} \mathrm{O}\right)$. Batimétricamente se encuentra desde el intermareal hasta los $150 \mathrm{~m}$ de profundidad (Bernard, 1983) y habita fondos de arenas gruesa a muy gruesa, conchíferos e incluso gravas y bolones, los cuales son característicos de ambientes altamente energéticos (Fundación Chinquihue, 2012; Jerez et al. 1999). Estos tipos de sustratos representan una fracción limitada del bentos en el mar interior de Chiloé, por lo cual es 
común encontrar bancos o parches discretos con altas densidades (Jerez et al. op. cit.). Alcanza una talla máxima de $39 \mathrm{~mm}$ de longitud (Osorio, 2002), transformándola en la almeja con importancia comercial de menor tamaño en las costas de Chile (Jerez et al. op. cit.).

La especie está sujeta a extracción formal a partir del año 2000 y según las estadísticas pesqueras, durante el año 2010 se reportó el desembarque máximo de la especie, el cual fue de 9.608 t. Actualmente esta pesquería es administrada a través de la figura de Plan de Manejo (ver SUBPESCA, 2013), sin embargo, desde el 2010 los desembarques han disminuido progresivamente, alcanzado un total de 3.070 t. en el 2016, lo que representa una baja del $68 \%$ respecto de lo reportado en el 2010 (SENAPESCA, 2016).

Se han esgrimido razones relacionadas con el mercado, para explicar la disminución en los desembarques. Sin embargo, un estudio realizado durante el 2014 - 2015 reportó que solo dos, de los ocho bancos prospectados mantenían una población potencialmente productiva en la zona. La disminución del desembarque fue atribuida tanto a la sobreexplotación del recurso, como a la no inclusión de la conectividad espacial de los distintos bancos locales en el manejo pesquero (Vega et al. 2016). Esta situación ya ha sido mencionada anteriormente para explicar la poca efectividad de las medidas administrativas pesqueras utilizadas en otras almejas en la costa de Chile (Aburto \& Stotz, 2013). Por lo que antes de la implementación de estrategias que apuntan a mejorar la gestión de los recursos pesqueros altamente variables, es necesario conocer la escala espacial dentro de la cual se conectan los diferentes bancos o poblaciones locales (Aburto \& Stotz, op. cit.).

Existen distintas aproximaciones para estudiar la dinámica de metapoblaciones, siendo una de ellas los modelos en función de la estructura del paisaje. Estos modelos relacionan propiedades a nivel de las especies (i.e. dispersión y colonización) con aquellas propiedades inherentes del paisaje (i.e. tamaño del parche y número y distribución espacial de los parches) para comprender la variación temporal de ocupación de estos (Etienne et al. 2004; Hanski \& Gyllenberg, 1997; MacPherson \& Bright, 2011), dando origen a la Teoría de la Metapoblación Espacialmente Realista (Ovaskainen \& Hanski, 2003).

Este nuevo enfoque podría aplicarse de manera eficiente en la determinación de la dinámica metapoblacional de un número finito de poblaciones locales, las cuales se caracterizan por presentar una alta variación en las cualidades intrínsecas de cada una de ellas. La variabilidad en la conectividad entre parches, se manifiesta en las diferencias observadas tanto en la ubicación espacial de los bancos, como en la migración dependiente de la distancia (Sale et al. 2006), por lo que en base a esta teoría, existen una serie de estudios teóricos y empíricos que han indagado es cómo la dinámica de la metapoblación es afectada por la capacidad de dispersión y colonización de las especies de acuerdo al número y distribución espacial de los parches (Mestre et al. 2016). Para esto, se han desarrollado una serie de herramientas realistas que permiten una estimación ajustada estadísticamente a los parámetros del modelo (Etienne et al. 2004; Sale et al. 2006), llegando a ser simulaciones altamente predictivas de la dinámica de colonización y recolonización de parches en el tiempo (Thomas \& Hanski, 1997; Wahlberg et al. 2001).

Dado que estos modelos no incluyen una descripción de la dinámica local de la población, han sido denominados como "Modelos Estocásticos de la Ocupación de Parches" o SPOM por sus siglas en inglés, logrado un equilibrio entre los requisitos empíricos de los datos y la capacidad de generalización de los modelos en distintas especies y contextos ecológicos (Grimm et al. 2008; Hanski, 2002; MacPherson \& Bright, 2011).

Por lo tanto, ante la disminución de las poblaciones de T. gayi en el Mar Interior de Chiloé, y al evidente grado de conectividad que existe o existió entre las poblaciones locales distribuidas en la zona, es necesario analizar el comportamiento de la dinámica metapoblacional para ayudar a definir las medidas que permitan la recuperación de las poblaciones.

En el presente trabajo se realizaron diversas simulaciones mediante la aplicación del modelo de Ocupación de Parches Estocástico propuesto por Hanski (1999), y el modelo de grado de incidencia, propuesto por Hanski (1994), para dar respuesta a las siguientes interrogantes: ¿cuáles son los efectos de la disminución del número de bancos locales, sobre la conectividad espacial de la población de $T$. gayi en el mar interior de Chiloé? y ¿Existen bancos que, en base a su ubicación y tamaño, inciden sobre la presencia de otros bancos distribuidos en la zona?. 


\section{MATERIALES Y MÉTODOS}

Área de estudio y entorno virtual del modelo

El área de estudio correspondió a la zona norte del Mar Interior de Chiloé, en la Región de Los Lagos, lugar en la que se encuentran distribuidos los bancos - parches de Tawera gayi descritos para la zona (Fundación Chinquihue, 2012; Jerez et al. 1999; SUBPESCA, 2013; Vega et al. 2016) (Fig. 1).

Para simular el rango de expansión de cada uno de los bancos, se utilizó el paquete MetaLandSim del software R 3.3.0 (Mestre et al. 2018; Mestre et al. 2016 b), el cual proporciona un entorno virtual que permite la experimentación y simulación de una serie procesos involucrados en la viabilidad de la metapoblación. Este paquete realiza los cálculos considerando dos escalas (i.e. paisaje y rango) (Peck et al. 2004; Zurell et al. 2009), lo que permitió simular la ocupación de la metapoblación en un paisaje dinámico, calculando además la métrica de dicha conectividad. Para el caso de la escala de rangos, el paquete permitió calcular la dispersión del modelo y la simulación del rango de expansión (Mestre et al. 2016 a).

El proceso de simulación se realizó en un paisaje virtual de 2250 hectáreas $(150 \mathrm{~km} \times 150 \mathrm{~km})$, lo que equivale al área del sector norte del mar interior de Chiloé donde se encuentran distribuidos los bancos utilizados actualmente en el Plan de Manejo. Dicha simulación se realizó en base a dos escenarios (Fig. 2):

1. Escenario 1, condición que fue considerada como inicial, antes de comenzar la pesquería, la cual contaba con 32 bancos, todos ocupados, y cuyas dimensiones y densidades fueron descritas por Jerez et al. (1999).

2. Escenario 2, condición que contaba con solo 8 bancos presentes en el área, lo cual se basa en lo descrito por Fundación Chinquihue (2012).

En ambos escenarios se consideró que las corrientes tienen una dirección que va desde sureste a nor-oeste, tal como ha sido sugerido por Vega et al. (2016).

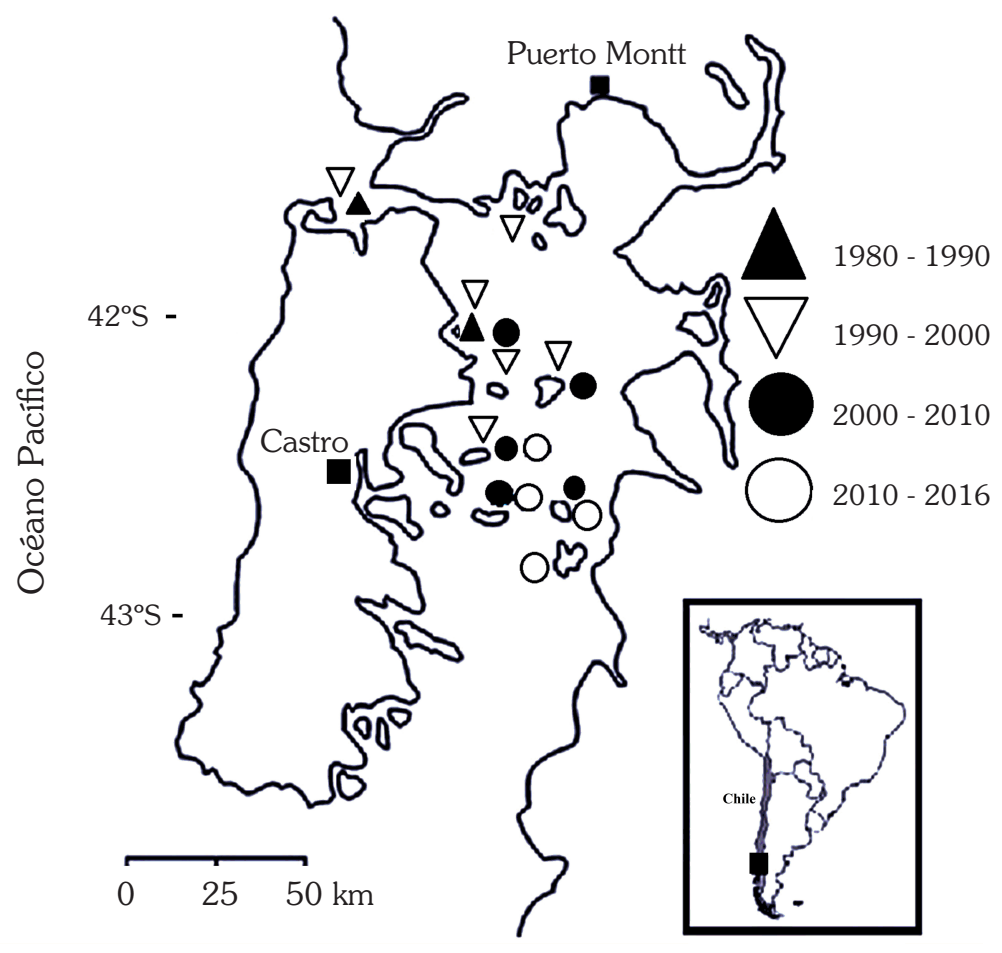

Fig. 1. Ubicación de los bancos explotados del recurso Juliana (Tawera gayi) en el mar interior de Chiloé entre 1980 al 2013. Las figuras representan la ubicación de los bancos descritos entre 1980 y el 2016 (i.e. Triangulo negro entre 1980 - 1990; triangulo blanco entre 1990 2000; circulo negro entre 2000 - 2010 y triangulo blanco entre 2010 - 2013). 
Impacto de la disminución del número de bancos sobre la conectividad

Para evaluar el impacto de la disminución del número de bancos o parches en la zona, se realizó una simulación de la dinámica del paisaje, siguiendo las indicaciones descritas por Mestre et al. (2016) a. Para esto se crearon paisajes aleatorios (representados en forma de gráficos) los cuales sufrieron cambios dinámicos, como la pérdida de parches y la pérdida de hábitat.

La representación gráfica resultante representó la conectividad según los escenarios descritos anteriormente.

La transición de un paisaje ocupado en el tiempo $1(t)$, a uno de tiempo $2(t+1)$ se consiguió mediante la función del Modelo de Ocupación de Parches Estocástico (SPOM), el cual simula el estado de ocupación de las especies en parches de hábitat (Moilanen, 2004) y enlaza la información biológica con la información de ocupación, lo que permitió la predicción de la probabilidad de extinción o de colonización de cada parche en un paisaje (Etienne et al. 2004). Para evaluar los modelos de ocupación en los escenarios propuestos según el modelo SPOM, se utilizaron los siguientes supuestos:

1) Dispersión definida mediante las ecuaciones de Kernel: " $\mathrm{D}(\mathrm{Dij}, \alpha)=\exp (-\alpha$.dij)" (Hanski, 1994, 1999).

2) Conectividad: " $\mathrm{Si}=$ sum(pj.D(dij,alpha). $\mathrm{Al} \wedge \mathrm{j}) "$ (Moilanen, 2004) donde $\mathrm{Ai}$ y $\mathrm{Aj}$ son las áreas de los parches (parche focal $=\mathrm{Ai}$ ) y otros parches $\mathrm{j}$, respectivamente y dij es la distancia entre parches i y j, y pj es el estatus i de ocupación del parche $\mathrm{j}(0 / 1)$.

3) Función de Colonización: $\mathrm{Ci}=\mathrm{Si}^{\wedge} 2 /$ $\left(\mathrm{Si}^{\wedge} 2+\mathrm{y}^{\wedge} 2\right)$ (Hanski, 1994, 1999), el cual es la primera versión de la probabilidad de colonización, e incluye el effect Alle, sin embargo, la fuerza de este efecto no puede ser modificado (Hanski (1994)). La probabilidad de colonización fue definida como una función sigmoidea de la conectividad del parche i.

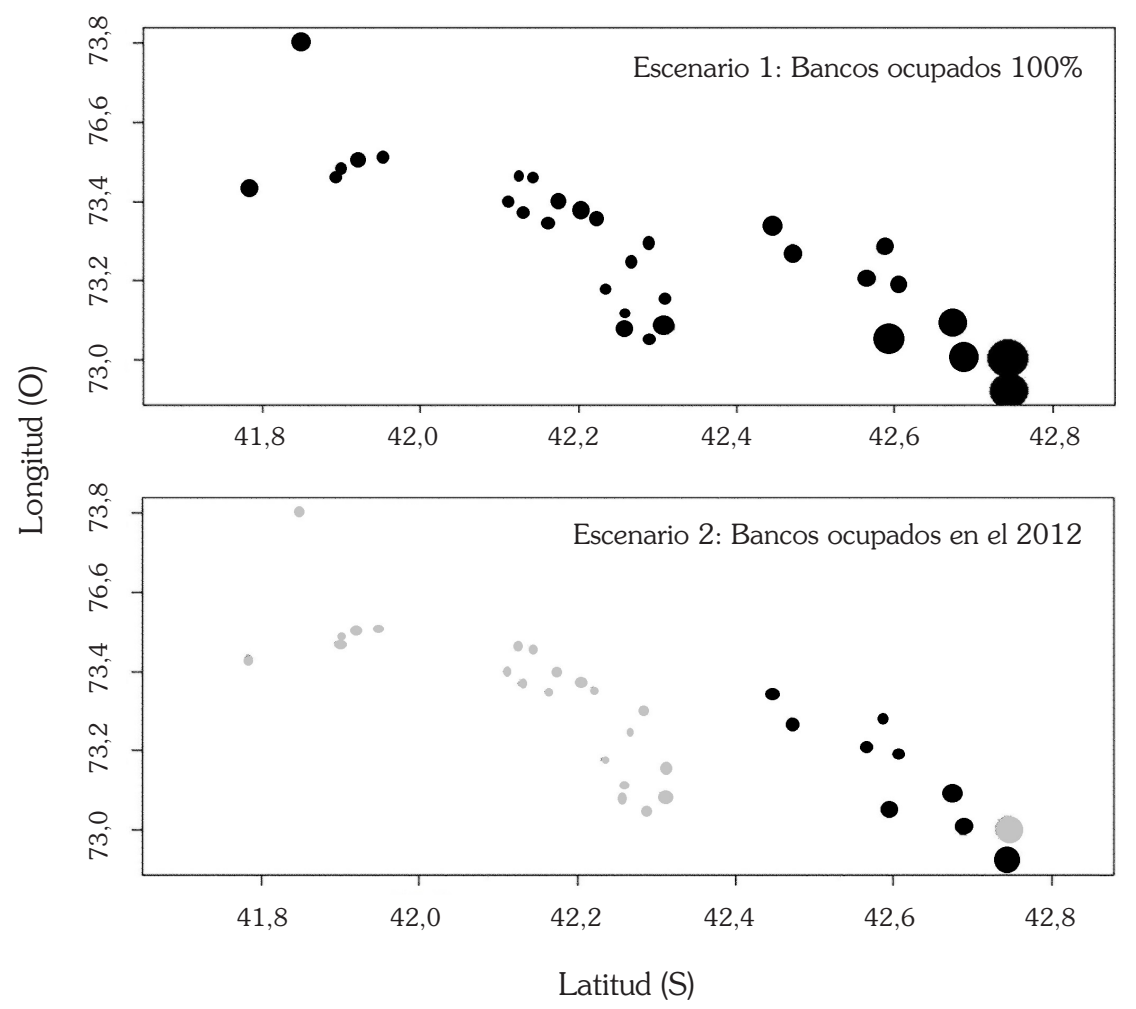

Fig. 2. Representación gráfica de los parches de la metapoblación ocupados en la zona del mar interior de Chiloé. Escenario 1 distribución de parches en el año 1999 según Jerez et al. (1999) B) Parches ocupados (círculos negros) y desocupados (círculos grises) en el año 2012 según Fundación Chinquihue et al. (2012). El tamaño del parche corresponde al área estimada del banco. 
4) Función de Extinción: definida por $\mathrm{Ei}=\min \left(1, e / \mathrm{Ai}^{\wedge} \mathrm{x}\right)($ Hanski, 1994, 1999).

El gráfico resultante fue el resultado de repetir 100 veces la simulación, para cada uno de los escenarios indicados. Los argumentos utilizados se especifican en la Tabla 1.

Evaluación del grado de incidencia de cada parche (Ji).

La aplicación del modelo de incidencia permitió obtener la probabilidad a largo plazo de que un fragmento esté ocupado (incidencia Ji), como resultado de la colonización $(\mathrm{Ci})$ y de la extinción (Ei). El modelo predice la probabilidad de equilibrio de ocupación de cada parche, como una función de la estructura espacial de toda la metapoblación, es decir en función de las ubicaciones y los tamaños de los parches.

La estimación de los parámetros se basó en la descripción realizada por Hanski (1999), y las sugerencias realizadas por Etienne et al. (2004). El método utilizado para determinar los parámetros fue el de "Regression of snapshot data", usando una sola instantánea (Oksanen, 2004), que utiliza el volumen, la estructura espacial y el estado de ocupación de cada uno de los bancos considerados en el modelo. Los parámetros utilizados fueron calculados en función de la información descrita por Jerez et al. (1999) para el escenario 1 y los parámetros resultantes incluidos en la simulación fueron:

Alfa $=3,3 \times 10^{-5}$, el cual relaciona la extinción con la distancia.

$\mathrm{x}=6,157579 \times 10^{-2}$, el cual determina el Parámetro de riesgo de extinción.

$y=1,324436 \times 10^{-5}$, el cual determina la probabilidad de colonización.

$e=9.582169 \times 10^{-1}$, parámetro que define la probabilidad de extinción en un parche de unidad de área.

Mientras que para el escenario 2 los parámetros fueron calculados a partir de la información de Fundación Chinquihue (2012) y cuyos valores fueron:

$$
\begin{aligned}
& \text { Alfa }=3,3 \times 10^{-5} \\
& x=1,648568 \\
& y=1,51308 \times 10^{6} \\
& e=1,5113748 \times 10^{2}
\end{aligned}
$$

Tabla 1. Argumentos, descripción y código utilizado en el desarrollo del modelo de Ocupación de Parches Estocástico (SPOM por sus siglas en inglés) propuesto por Hanski (1999) y desarrollado a través del paquete MetaLandSim del software R.3.3. (Mestre et al. 2014, 2016a, b y 2017).

\begin{tabular}{llc}
\hline \multicolumn{1}{c}{ Argumentos } & \multicolumn{1}{c}{ Descripción } & Código utilizado \\
\hline iter & Número de repeticiones de iteración & 100 \\
\hline mapsize & Tamaño del paisaje en metros & 150000 \\
\hline dist_m & Distancia media entre parches & 0,01207315 \\
\hline Meanarea & Área Media (Ha) & 37,67 \\
\hline sd & Desviación estándar del área & 23,7 \\
\hline Npatch & Numero de parches & Scenario 1: 32 \\
\hline disp & Dispersión (m) & Scenario 2: 8 \\
\hline span & Número de años simulados & 30000 \\
\hline par1 & Creación simultánea y destrucción de parches & 15 \\
\hline par2 & Parámetro creación & stoc \\
\hline Par3 & Parámetro de destrucción & northerndness \\
\hline method & Porcentaje de parches ocupados por la especie & southerndness \\
\hline parm & ocurrencia & porcentaje \\
\hline nsew & Punto de entrada de la especie al paisaje & 50 \\
\hline succ & Estado biológico de la llegada de la especie (Juliana= larvas) & E (Este) \\
\hline
\end{tabular}


En ambos escenarios no se considera el efecto de la extracción, y se asumió que una vez iniciada la simulación la extracción de recursos es cero.

\section{RESULTADOS}

La simulación de la conectividad espacialmente explícita para los bancos de la almeja Tawera gayi del mar interior de Chiloé (Fig. 3) nos reveló que la dispersión mínima para lograr una conexión de más del $80 \%$ de los bancos en el escenario 1, se logró a los 30 km de separación entre bancos, mientras que para lograr la conectividad en el segundo escenario (i.e. solo 8 bancos presentes) se logró a los $20 \mathrm{~km}$, pero con una disminución importante del área de dispersión. Por lo tanto, el área de cobertura para la probabilidad de expansión de la especie en la zona disminuyó hasta en $2 / 3$, al reducirse el número de bancos o parches presentes.

En cuanto a la incidencia que cada uno de los parches tuvo sobre la dinámica metapoblacional (Fig. 4), se observó que para el caso del Escenario 1,
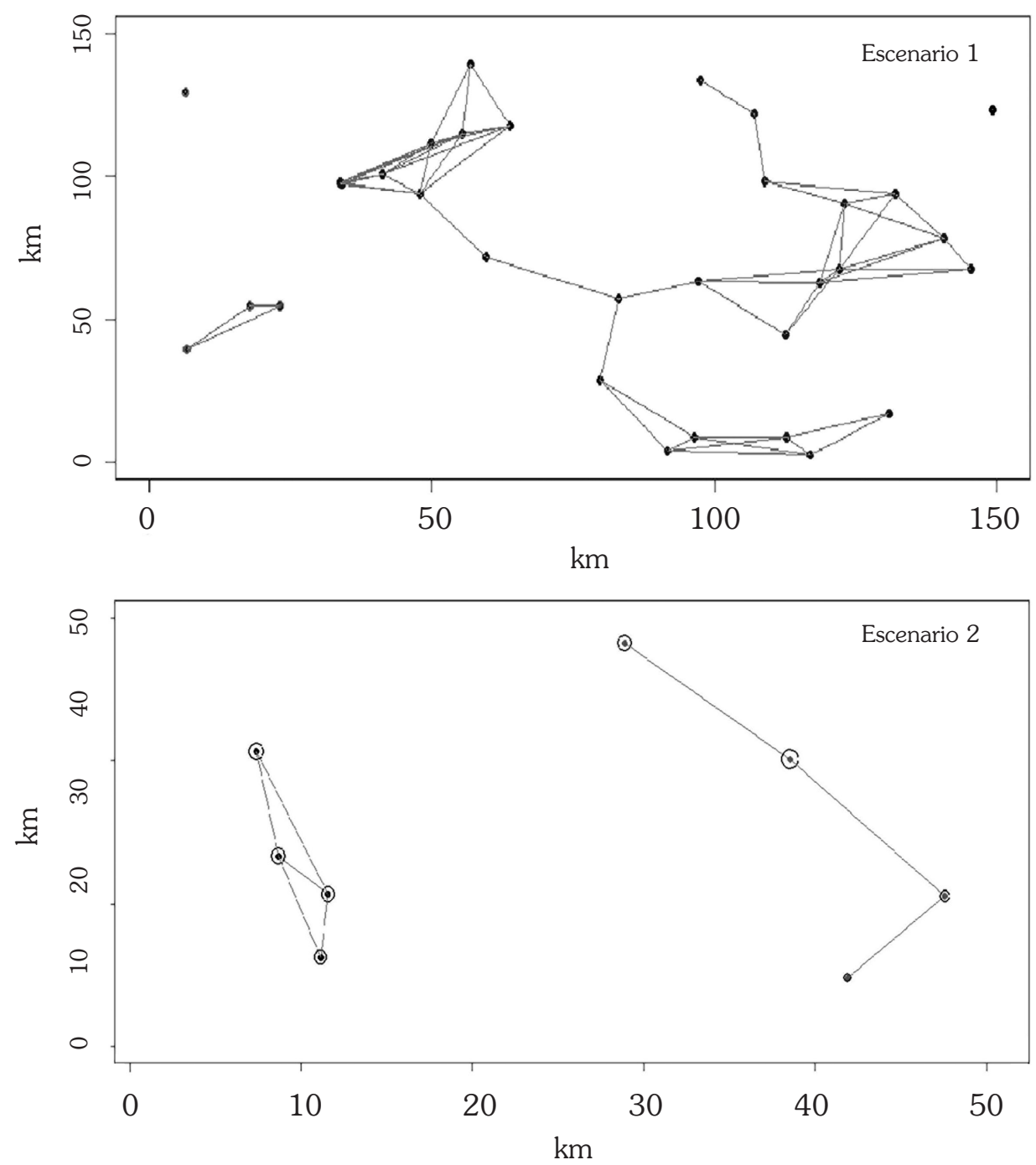

Fig. 3. Representación gráfica de la conectividad entre bancos para los dos escenarios propuestos. Escenario 1: 32 bancos y un área de $150 \mathrm{~km}^{2}$, con $30 \mathrm{~km}$ de dispersión. B) Escenario de 8 bancos distribuidos en $50 \mathrm{~km}^{2}$, con $20 \mathrm{~km}$ de dispersión. 
todos los bancos inciden en el banco más cercano. Sin embargo, al disminuir el número de parches ocupados a un $25 \%$ en el Escenario 2, se observó una pérdida de la mayoría de los bancos ubicados hacia el Nor-oeste, aumentando la incidencia de los bancos ubicados en la dirección contraria (Sureste).

En relación a la simulación de ocupación del paisaje (Fig. 5), se observó que con una tasa de ocupación del $25 \%$ cada 10 años, los escenarios propuestos se ajustan a lo reportado en la zona $y$ de mantenerse las condiciones de pérdida de conectividad entre bancos, el modelo predice que solo dos parches sobrevivirían a un tiempo 3 , lo cual es coincidente con el modelo de incidencia mencionado anteriormente.

Los resultados de la simulación del Modelo Estocástico de Ocupación de Parches para cada uno de los escenarios propuestos en un periodo de 15 años (Fig. 6), mostraron que en relación al área promedio de los bancos, estos diferían diametralmente entre ambos escenarios, ya que mientras en el escenario 1 se observa un incremento del área por parche en los primeros 7 años, en el escenario 2 este disminuye.

La distancia promedio del banco más cercano, presentó en el escenario 2, un aumento del doble que la distancia promedio determinada para el escenario 1. Sin embargo, al observar el número de bancos para cada escenario se pudo apreciar que estos se mantuvieron en el tiempo.

Finalmente, el porcentaje de ocupación de hábitat, o en otras palabras el porcentaje de ocupación de los parches reportados, escenario 2 se vio una fuerte caída en la ocupación de los parches, llegando incluso a valores cercanos a cero después de 8 años de simulación.

\section{DISCUSIÓN}

La variabilidad de las abundancias poblacionales que están sujetas a explotación comercial, son altamente sensibles a la dinámica del reclutamiento, lo cual se debe a la relación que

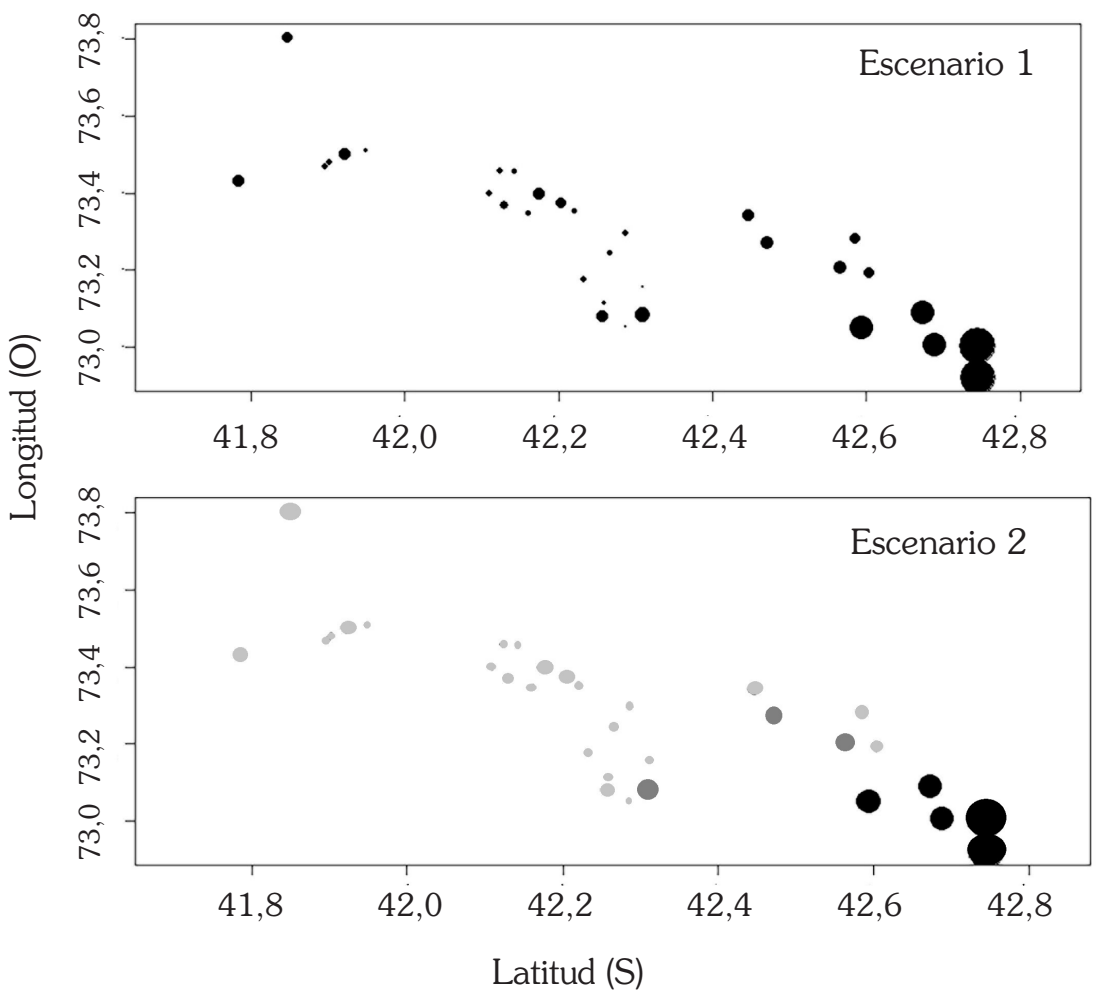

Fig.4. Representación gráfica del modelo de Incidencia de ambos escenarios ( $1=32$ bancos; $100 \%$ de éxito de los bancos; $2=8$ bancos; $25 \%$ de éxito de los bancos). A mayor tamaño e intensidad del color negro, mayor es la incidencia del banco en la metapoblación. 

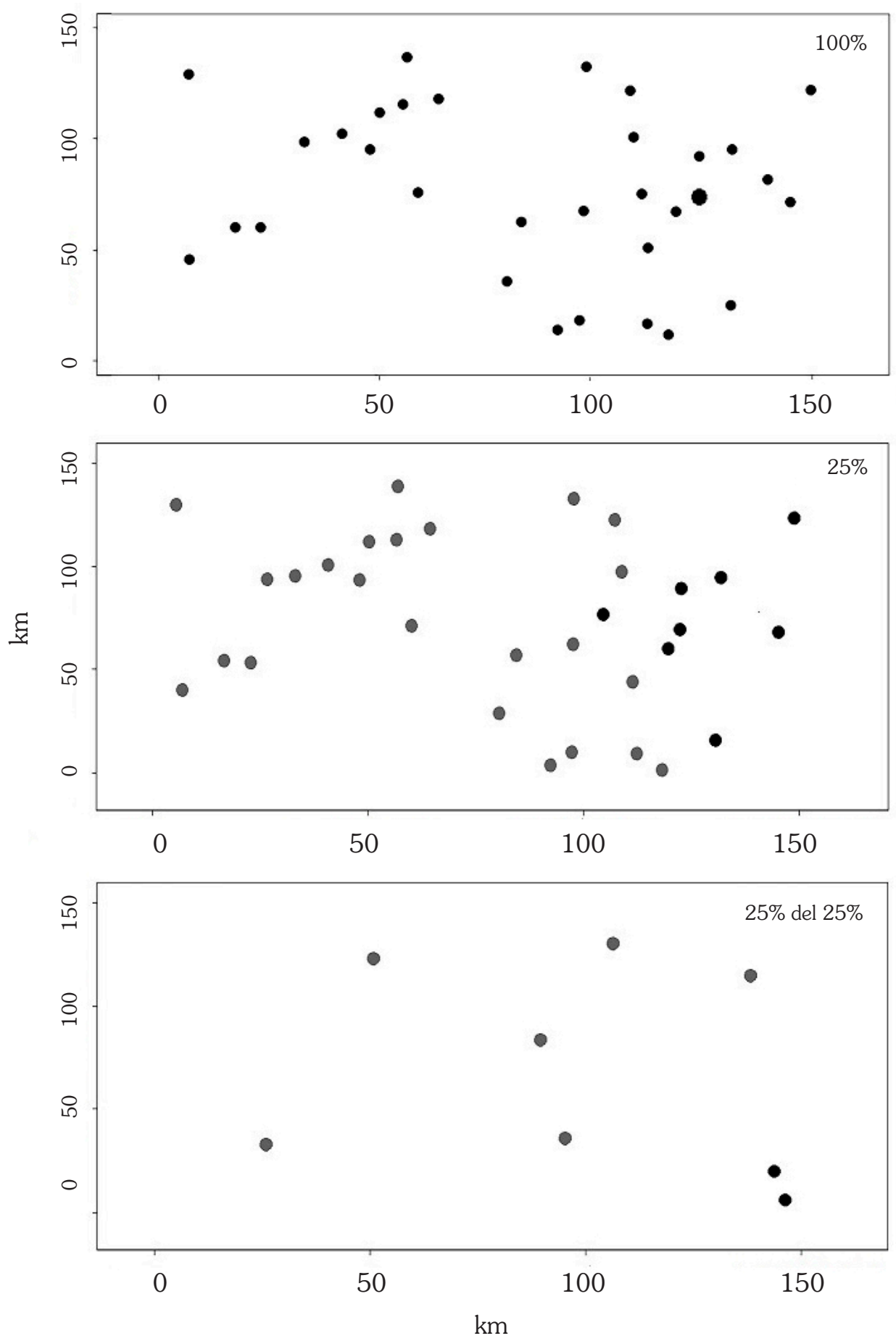

Fig. 5. Simulación gráfica de la ocupación del paisaje de los bancos de juliana bajo tres situaciones A) 32 bancos con 100\% de ocupación, B) 32 bancos con 25\% de ocupación y

C) 8 bancos con $25 \%$ de ocupación. 


\title{
Escenario 1
}

\author{
Escenario 2
}

Área promedio
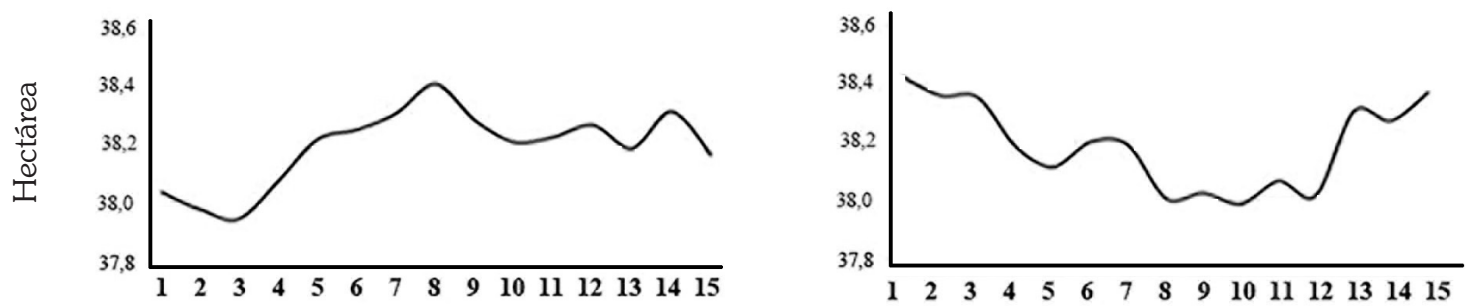

Distancia promedio del banco más cercano
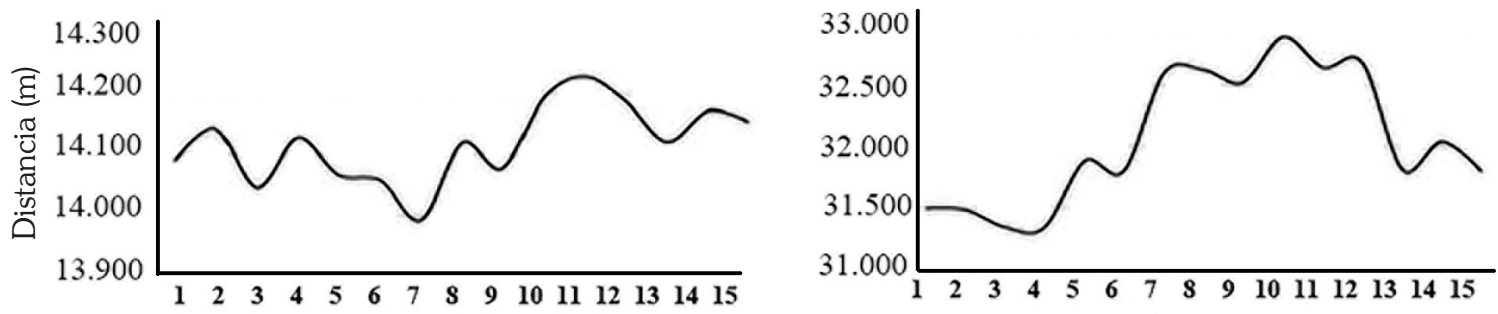

Número de bancos
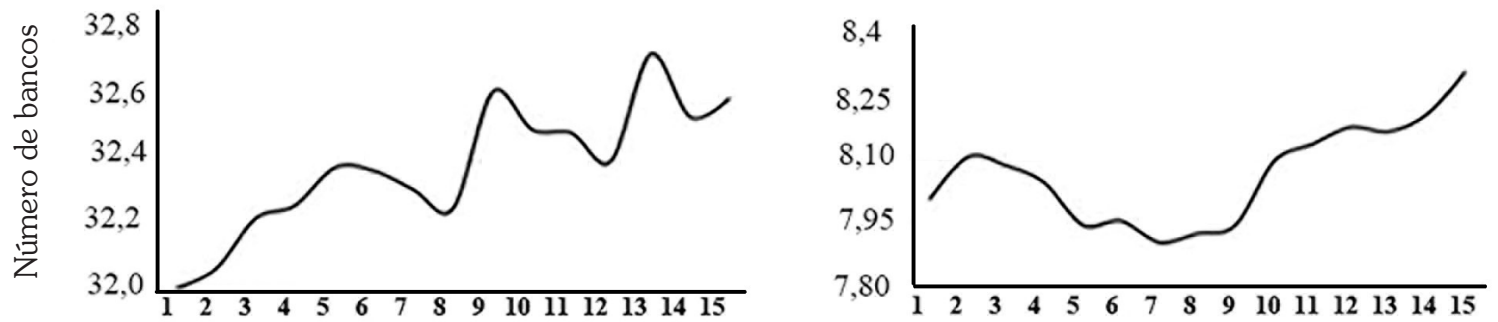

Ocupación del hábitat
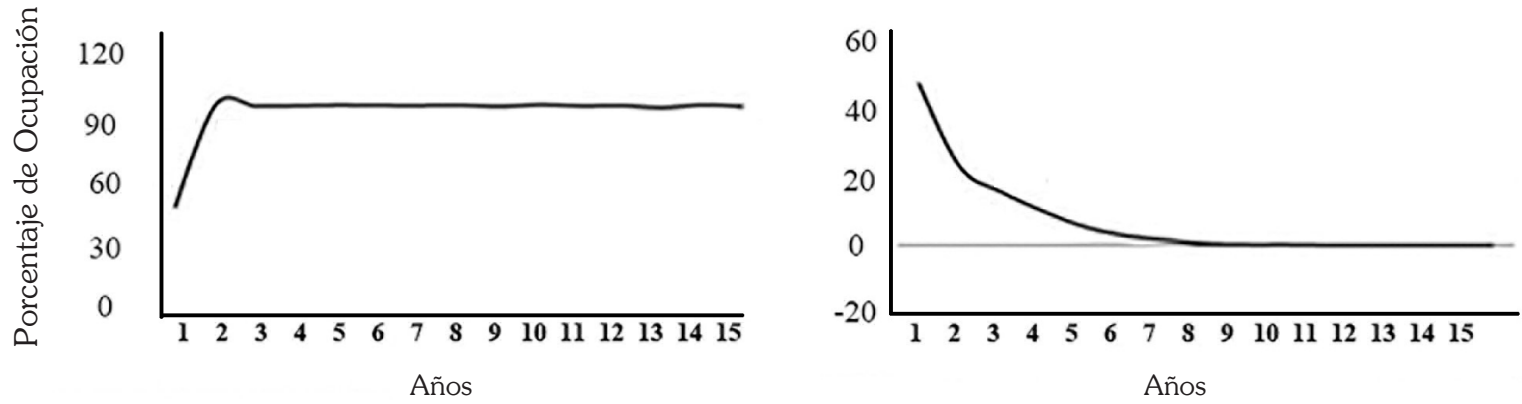

Fig. 6. Resultado de la simulación resultante del Modelo Estocástico de Ocupación de Parches para para los dos escenarios durante 15. Los gráficos son el resultado de 100 iteraciones. 
existe entre el suministro de larvas y la biomasa reproductiva de la población, la que a su vez depende de la actividad pesquera (Bakun, 1996; Hsieh et al. 2006). Ante esto, es válido pensar que el éxito del reclutamiento es una función de la abundancia de los reproductores (Myers, 1997), por lo que el suministro larval juega un rol fundamental en la sobrevivencia de las poblaciones locales, y que en el caso de especies marinas sésiles o de poca movilidad suele ser el mecanismo que asegura la conectividad entre poblaciones locales distanciadas entre sí. Por ejemplo, en la almeja Spisula solidissima en la costa noreste de Estados Unidos, se ha visto que el número de larvas liberadas en una región particular, afecta el asentamiento en las regiones contiguas (Zhang et al. 2015).

Es esperable entonces, que la disminución de larvas provoque un efecto directo en los procesos de dispersión y colonización de los bivalvos bentónicos, ya que la extinción local y la recolonización se sustenta por el movimiento de individuos entre zonas (Hanski, 1997). En el presente trabajo esto quedaría de manifiesto con la disminución del área de dispersión observada para los bancos de la almeja Juliana, lo que impacta directamente en el área de cobertura de la probabilidad de expansión. Si la tasa de colonización disminuye, es esperable que el grado de aislamiento de los bancos locales (distancia entre grupos vecinos) aumente y genere una pérdida de conectividad, tal como se observa en la simulación realizada para $T$. gayi.

Al perderse la conectividad, las poblaciones locales actúan de manera independiente, originando la sobrevivencia de unos pocos bancos completamente desconectados. Bidegain et al. (2013), describe un patrón similar en la especie Ruditapes decussatus en la costa de España, donde los procesos que explican la sobrevivencia de la especie estarían asociados a un "autoreclutamiento" de los bancos locales. En el mismo sentido Tezuka et al. (2012) reporta que la variabilidad de la abundancia de la almeja Ruditapes philippinarum en Japón, estaba influenciada por los procesos que ocurren después del asentamiento inicial y no por la llegada de nuevas larvas, lo que origina una asincronía entre las poblaciones locales. Esto constituye otro de los factores claves para la persistencia de una metapoblación, ya que cuando las poblaciones locales se hacen independientes, la probabilidad de extinción disminuye (Smith \& Smith, 2015).

El aislamiento provoca que el tamaño de la población local juegue un rol preponderante en la sobrevivencia de la especie (Dunham \& Rieman, 1999), provocando que esta dependa de las poblaciones de mayor tamaño. Hábitats disponibles pueden sustentar poblaciones grandes, las cuales son menos vulnerables a la extinción debido a factores demográficos estocásticos (Lande, 1993), lo que explica el aumento de la incidencia de los bancos de la Isla Desertores en el escenario 2 y su influencia en los bancos ubicados en las inmediaciones.

Al mantenerse la extracción de la fracción reproductiva de la especie, la población se deprime a tal manera que solo es posible la sobrevivencia de 2 de los 32 bancos iniciales a 15 años, lo cual coincide con lo observado por Vega et al. (2016) en la zona de estudio. Esto ya ha sido observado en otras especies de bivalvos sujetas a explotación comercial alrededor del mundo, por ejemplo, Peterson (2002) determinó que el reclutamiento de la almeja Mercenaria mercenaria en las zonas de pesca de Carolina del Norte disminuyó al aumentar la presión de extracción, provocando la disminución de hasta un 95\% de la abundancia poblacional en menos de 11 años.

$\mathrm{Al}$ agregar otros factores que acrecienta aún más la disminución del tamaño del hábitat, es esperable que las poblaciones difícilmente logren una conectividad, ya que un hábitat pequeño mantiene poblaciones reducidas (Kindvall \& Ahlen, 1992; Kindvall, 1996). Por ejemplo, es conocido el rol que tiene la textura de los sedimentos en el éxito de la colonización de bivalvos de fondos sedimentarios (Urban, 1994), restringiendo las estrategias de expansión y sobrevivencia de la metapoblación. En este sentido Pulliam (1998) sugiere que cuando una población local cuenta con condiciones óptimas de hábitat, estas pueden ser capaces de producir una gran cantidad de larvas, por lo que la disponibilidad del hábitat adecuado para metamorfosearse es un factor clave en la sobrevivencia de la especie (Bidegain et al. 2013). $\mathrm{Si}$ consideramos que la almeja Juliana habita ambientes específicos (i.e. con sedimentos gruesos y altamente energéticos) (Fundación Chinquihue, 2012; Jerez et al. 1999), podemos presumir que no existe una disponibilidad de hábitat que permita 
la rápida recuperación de la especie y que queda de manifiesto con la ubicación de los bancos reportados por Jerez et al. (op. cit.) al momento de iniciarse la explotación. Al no existir una dispersión mayor, producto de la disminución de larvas en el sistema, y que no exista gran cantidad de espacios óptimos para ser colonizados debido a la preferencia de un tipo de sustrato, podemos comprender por qué en la simulación no aumenta, ni en el número de bancos colonizados, ni en el área de los parches de Juliana.

Adicionalmente, al analizar los resultados en su conjunto en una perspectiva temporal mayor, queda de manifiesto que la extracción de la especie originó una alternancia entre los fenómenos de gran conectividad y auto-reclutamiento, lo que en primera instancia permitía asegurar la sobrevivencia de la especie.

Por muchos años se consideró la existencia de estas dos estrategias por separado, y ante esto los esfuerzos del manejo pesquero se enfocaron en categorizar las poblaciones entre fuentes y sumideros, considerando incluso que al proteger la población fuente o madre, se aseguraba la sustentabilidad de la especie (Wilberg et al. 2008). Sin embargo, teóricamente es posible que la alternancia de estos procesos sea más bien un efecto compensatorio sobre la probabilidad de ocupación del espacio por parte de las metapoblaciones (Thomas \& Jones, 1994). En otras palabras, al existir mayor conectividad se compensa la reducción del espacio, por medio de un mayor número de parches colonizados; mientras que, al reducirse la colonización, aumenta el espacio disponible dentro del banco, disminuyendo la probabilidad de extinción, lo que compensa su aislamiento. Ante la existencia de estas alternancias de estrategias, ninguna población local puede ser capaz de asegurar la supervivencia a largo plazo de una especie dada, sino que más bien parece ser que el efecto combinado de muchas poblaciones es capaz de lograr un resultado exitoso (Defeo \& Cansado, 2015). Ante esto, debemos considerar que no existe un único factor que pueda provocar la extinción local de las especies, sino que más bien es la concatenación de distintos factores que forman una sinergia entre sí.

Con estos resultados podemos concluir que la disminución del número de bancos afectó seriamente la conectividad de la especie, originando un cambio en la dinámica de la metapoblación, pasando de una estrategia basada en un conjunto de parches altamente conectados, a otra cuya conectividad es reducida, originando un aumento en la importancia de los procesos de "auto-reclutamiento" de la población.

Adicionalmente, existen evidencias que, además de la conectividad larval, otros factores pueden afectar la sobrevivencia de las poblaciones locales, entre las que se cuentan una pobre supervivencia de juveniles y un crecimiento lento después del asentamiento de las larvas (Quijón et al. 2007; Zhang et al. 2015). De continuar la extracción del recurso, sobre estas poblaciones desconectadas, claramente se pone en riesgo la sostenibilidad no solo de la actividad pesquera, sino que la sobrevivencia de la especie en la zona, demostrando la importancia de considerar la conectividad espacial en el manejo pesquero. Además es necesario considerar los efectos sinérgicos que la extracción de especies de interés comercial pueda generar en los ecosistemas marinos, evitando caer en una mirada reduccionista - determinista clásica.

\section{AGRADECIMIENTOS}

Resultados parciales del presente trabajo fueron parte del proyecto FIP 2014-20 "Evaluación directa del recurso Juliana (Tawera gayi) en el mar interior de Chiloé e Islas Desertores, Región de Los Lagos". Los autores agradecen el apoyo de los investigadores del Instituto de Fomento Pesquero señores Luis Ariz, Claudio Vega, Lianella Diaz y Elías Pinilla. Este trabajo fue presentado en el XXXVII Congreso de Ciencias del Mar realizado en Valparaíso.

\section{LITERATURA CITADA}

Aburto, J., \& Stotz, W. (2013). Learning about TURFs and natural variability: Failure of surf clam management in Chile. Ocean and Coastal Management, 71, 88-98.

Bakun, A. (1996). Patterns in the Ocean. Ocean processes and marine population dynamics. ( $1^{\mathrm{a}}$ edn.). California Sea Grant, in cooperation with Centro de Investigaciones Biológicas del Noroeste, La Paz, México. 
Bernard, F. R. (1983). Catalogue of the Living (Bivalvia of the Eastern Pacific Ocean: Bering Strait to Cape Horn). Canadian Special Publication of Fisheries and Aquatic Sciences, Vol. 61: 112pp.

Bidegain, G., Bárcena, J. F., García, A., \& Juanes, J. A. (2013). LARVAHS: Predicting clam larval dispersal and recruitment using habitat suitability-based particle tracking model. Ecological Modelling, 268, 78-92.

Botsford, L. W. (2001). Physical influences on recruitment to California current invertebrate populations on multiple scales. ICES Journal of Marine Science, 58(5), 1081-1091.

Caddy, J. F., \& Defeo, O. (2003). Enhancing or restoring the productivity of natural populations of shellfish and other marine invertebrate resources. Fisheries Technical Paper. No. 448. Roma. Retrieved from http://www.fao.org/docrep/006/y5029e/ y5029e00.htm Accessed 27 March 2018.

Defeo, O., \& Cansado, S. (2015). The relevance of the metapopulation concept to fisheries: a review. Marine Stewardship Council Science Series, 3(1), 1-80.

Dell, R. (1974). Antarctic and Subantarctic mollusca: Amphineura, Scaphopoda and Bivalvia. Discovery Report, 33, 93-250.

Dunham, J. B., \& Rieman, B. E. (1999). Metapopulation structure of Bull Trout: Influence of Physical, Biotic and Geometircal Landscape Characteristics. Ecological Applications, 9(2), 642-655.

Etienne, R. S., ter Braak, C. J. F., \& Vos, C. C. (2004). Application of Stochastic Patch Occupancy Models to Real Metapopulations. Ecology, Genetics and Evolution of Metapopulations, 105-132.

Fundación Chinquihue. (2012). Evaluación del estado de situación del recurso Juliana (Tawera gayi, Hupe 1854 ) en bancos de Chiloé interior, Región de los Lagos. Subsecretaria de Pesca y Acuicultura, Mayo 2012, 1-15. http://www.subpesca.cl/ portal/618/articles-4788_informe.pdf

Grimm, N. B., Foster, D., Groffman, P., Grove, J. M., Hopkinson, C. S., Nadelhoffer, K. J., ... Peters, D. P. C. (2008). The changing landscape: Ecosystem responses to urbanization and pollution across climatic and societal gradients. Frontiers in Ecology and the Environment, 6(5), 264-272.

Hanski, I. (1994). A practical Model of Metapopulation Dynamics. The Journal of Animal Ecology, 1, 151-162.

Hanski, I. (1997). Metapopulation Dynamics. From Concepts and Observations to Predictive Models. In I. Hanski \& M. E. Gilpin (Eds.), Metapopulation Biology: Ecology, Genetics, and Evolution (pp. 6991). Londres: Academic Press Editors.

Hanski, I. (1999). Metapopulation ecology. (1 edn.). Oxford University Press Inc.

Hanski, I. (2002). In the midst of ecology, conservation, and competing interests in the society. Annales Zoologici Fennici, 39(3), 183-186.

Hanski, I., \& Gaggiotti, O. (2004). Metapopulation Biology. Past, Present, and Future. Metapopulation Biology: Ecology, Genetics, and Evolution (pp. 3-22). Londres: Academic Press Editors.

Hanski, I., \& Gilpin, M. E. (1991). Metapopulation dynamics: brief history and conceptual domain. Biological Journal of the Linnean Society, 42(1-2), https:// doi.org/10.1111/j.1095-8312.1991. tb00548.x

Hanski, I., \& Gilpin, M. E. (1997). Part III Metapopulation processes. Metapopulation Biology: Ecology, Genetics, and Evolution (pp. 211-214). Londres: Academic Press Editors. https://doi.org/http:// dx.doi.org/10.1016/B978-0123234452/50013-4

Hanski, I., \& Gyllenberg, M. (1997). Uniting two general patterns in the distribution of species. Science, 275(5298), 397-400.

Hsieh, C. H., Reiss, C. S., Hunter, J. R., Beddington, J. R., May, R. M., \& Sugihara, G. (2006). Fishing elevates variability in the abundance of exploited species. Nature, 443(7113), 859-862.

Jerez, G., Barahona, N., Ojeda, V., Brown, D., Osorio, C., Olguin, A., \& Orensanz, J. (1999). Estudio biológico pesquero de los recursos Tawera (Tawera gayi) y Culengue 
(Gari solida) en la X Región. Informe Final Proyecto Fondo de Investigación Pesquera, FIP:97-29. Valparaíso-Chile. http://www. subpesca.cl/fipa/613/articles-89615_ informe_final.pdf Accessed 27 March 2018.

Kindvall, O. (1996). Habitat heterogeneity and survival in a bush cricket metapopulation. Ecology, 77(1), 207-214.

Kindvall, O., \& Ahlen, I. (1992). Geometrical Factors and Metapopulation Dynamics of the Bush Cricket, Metrioptera bicolor Philippi (Orthoptera: Tettigoniidae). Conservation Biology, 6(4), 520-529.

Kininmonth, S., Beger, M., Bode, M., Peterson, E., Adams, V. M., Dorfman, D., Possingham, H. P. (2011). Dispersal connectivity and reserve selection for marine conservation. Ecological Modelling, 222(7), 1272-1282.

Lande, R. (1993). Risks of Population Extinction from Demographic and Environmental Stochasticity and. The American Naturalist, 142(6), 911-927.

Levins, R. (1969). Some Demographic and Genetic Consequences of Environmental Heterogeneity for Biological Control. Bulletin of the Entomological Society of America, 15, 237-240.

MacPherson, J. L., \& Bright, P. W. (2011). Metapopulation dynamics and a landscape approach to conservation of lowland water voles (Arvicola amphibius). Landscape Ecology, 26(10), 1395-1404.

Mestre, F., Cánovas, F., Pita, R., Mira, A., \& Beja, P. (2016)a. An $\mathrm{R}$ package for simulating metapopulation dynamics and range expansion under environmental change. Environmental Modelling and Software, 81, 40-44.

Mestre, F., Canovas, R., Pita, R., Mira, A., \& Beja, P. (2016) b. Model Parameter Estimation. https://dspace.uevora.pt/ rdpc/bitstream/10174/21118/5/ parametrization.pdf Accessed 27 March 2018.

Mestre, F., Canovas, F., Pita, R., Mira, A., \& Beja, P. (2018). Landscape Occupation Simulation in Dynamic Landscapes. https://cran.rproject.org/web/packages/MetaLandSim/ vignettes/landscape_simulation-knitr.pdf
Accessed 27 March 2018.

Moilanen, A. (2004). SPOMSIM: Software for stochastic patch occupancy models of metapopulation dynamics. Ecological Modelling, 179(4), 533-550.

Myers, R. A. (1997). Comment and reanalysis: paradigms for recruitment studies. Canadian Journal of Fisheries and Aquatic Sciences, 54(4), 978-981.

Oksanen, J. (2004). Incidence function model in R. Retrieved from http://cc.oulu.fi/ jarioksa/ opetus/openmeta/metafit.pdf Accessed 27 March 2018.

Osorio, C. (2002). Moluscos Marinos de importancia económica ( $1^{\mathrm{a}}$ edn.). Santiago, Chile: Editorial Salesianos.

Ovaskainen, O., \& Hanski, I. (2003). The speciesarea relationship derived from speciesspecific incidence functions. Ecology Letters, 6(10), 903-909.

Peck, L. S., Webb, K. E., \& Bailey, D. M. (2004). Extreme sensitivity of biological function to temperature. Functional Ecology, 18, 625630.

Peterson, C. H. (2002). Recruitment overfishing in a bivalve mollusc fishery: hard clams (Mercenaria mercenaria) in North Carolina. Canadian Journal of Fisheries and Aquatic Sciences, 59(1), 96-104.

Pineda, J., Hare, J., \& Sponaugle, S. (2007). Larval Transport and Dispersal in the Coastal Ocean and Consequences for Population Connectivity. Oceanography, 20(3), 22-39.

Pulliam, D. W. (1998). Sources, Sinks, and Population Regulation. American Society of Naturalists. https://doi. org/10.2307/2678832

Quijón, P. A., Grassle, J. P., \& Rosario, J. M. (2007). Naticid snail predation on early postsettlement surfclams (Spisula solidissima) on the inner continental shelf of New Jersey, USA. Marine Biology, 150(5), 873-882.

Rodriguez, S. R., Ojeda, F. P., \& Inestrosa, N. C. (1993). Settlement of benthic marine invertebrates. Marine Ecology Progress Series, 97(2), 193-207.

Sale, P. F., Hanski, I., \& Kritzer, J. (2006). The merging of metapopultion theory and marine ecology: establishing the historical 
context. Marine Metapopulations, (1967), 3-28.

Servicio Nacional de Pesca, SERNAPESCA. (2016). Anuario Estadístico de Pesca. Servicio Nacional de Pesca y Acuicultura. Valparaíso-Chile.

Smith, T. M., \& Smith, R. L. (2015). Elements Ecology (9th ed.). Pearson.

Snelgrove, P. V. R., \& Butman, C. A. (1994). Animal Sediment Relationships Revisited - Cause Versus Effect. Oceanography and Marine Biology, 32(January 1994), 111177.

Soot-Ryen, T. (1959). Pelecypoda. Acta Universitatis Lundensis II, 55, 1-87.

SUBPESCA. (2013). Propuesta de plan de manejo recurso Juliana Tawera gayi Región de Los Lagos. Subsecretaria de Pesca y Acuicultura, pp. 34. Valparaíso-Chile. http://www.aqua. cl/wp-content/uploads/sites/3/2013/11/ Plan-de-Manejo-juliana.pdf

Tezuka, N., Kamimura, S., Hamaguchi, M., Saito, H., Iwano, H., Egashira, J., Nakagawa, K. (2012). Settlement, mortality and growth of the Asari clam (Ruditapes philippinarum) for a collapsed population on a tidal flat in Nakatsu, Japan. Journal of Sea Research, 69, 23-35.

Thomas, C. D., \& Hanski, I. (1997). Butterfly Metapopulations. Metapopulation Biology: Ecology Genetics and Evolution, 359-386.

Thomas, C. D., \& Jones, T. M. (1994). Partial recovery of a skipper butterfly (Hesperia comma) from population refuges: lessons for conservation in a fragmented landscape. Journal of Animal Ecology, 62(3), 472481.

Urban, H.-J. (1994). Adaptations of six infaunal bivalve species of Chile: Coexistence resulting from differences in morphology, burrowing depth and substrate preference. Archive of Fishery and Marine Research, 42(2), 183-193.

Vega, C., Figueroa-Fábrega, L., Ariz, L., Canales, C., Romero, P., \& Pinilla, E. (2016). Evaluación directa del recurso Juliana Tawera gayi en el Mar Interior de Chiloé e Islas Desertores, Región de los Lagos. Informe Final Proyecto Fondo de Investigación Pesquera, FIP:2014-20. Valparaíso-Chile. http://www.subpesca.cl/ fipa/613/articles-89378_informe_final.pdf Accessed 27 March 2018.

Wahlberg, N., Kullberg, J., \& Hanski, I. (2001). Natural history of some Siberian melitaeine butterfly species (Nymphalidae: Melitaeini) and their parasitoids. Entomologica Fennica, 12(2), 72-77.

Wilberg, M. J., Irwin, B. J., Jones, M. L., \& Bence, J. R. (2008). Effects of source-sink dynamics on harvest policy performance for yellow perch in southern Lake Michigan. Fisheries Research, 94(3), 282-289.

Zhang, X., Haidvogel, D., Munroe, D., Powell, E. N., Klinck, J., Mann, R., \& Castruccio, F. S. (2015). Modeling larval connectivity of the Atlantic surfclams within the Middle Atlantic Bight: Model development, larval dispersal and metapopulation connectivity. Estuarine, Coastal and Shelf Science, 153, 38-53.

Zurell, D., Jeltsch, F., Dormann, C. F., \& Schröder, B. (2009). Static species distribution models in dynamically changing systems: How good can predictions really be? Ecography, 32(5), 733-744. 
